\title{
Anchor Variable Name
}

National Cancer Institute

\section{Source}

National Cancer Institute. Anchor Variable Name. NCI Thesaurus. Code C117299.

A reference to the CDISC date variable name that provides the start point from which the planned assessment schedule is measured. 